\title{
Assessing the Prevalence of Malaria, in the Case of Sigmo Health Center, Sigmo Woreda, Jimma Zone, South West Ethiopia
}

\author{
Guta Waktole Weyesa \\ Ethiopian Biodiversity Institute (EBI), Addis Ababa, Ethiopia
}

Email address:

waaqtoleegutaa@gmail.com

\section{To cite this article:}

Guta Waktole Weyesa. Assessing the Prevalence of Malaria, in the Case of Sigmo Health Center, Sigmo Woreda, Jimma Zone, South West Ethiopia. American Journal of Life Sciences. Vol. 9, No. 2, 2021, pp. 36-41. doi: 10.11648/j.ajls.20210902.13

Received: February 2, 2021; Accepted: April 27, 2021; Published: May 14, 2021

\begin{abstract}
Despite considerable efforts made throughout the century to eradicate or control malaria it is still the most prevalent and most devastating disease in the global tropics. The disease has a negative effect on the economic growth and perpetuates vicious cycles of poverty especially in the developing parts of the world. It costs Africa US $\$ 10-12$ billion every year in lost domestic product even though it could be controlled for a fraction of that sum. In Africa, the malaria causes about $20 \%$ of cerebral conditions leading to coma and death. One important strategy to prevent malaria infection is the use of insecticide treated mosquito net. Many researches were done in Ethiopia on the prevalence of malaria. Even though many researches were done on the prevalence of malaria in other place of the country, no any recent study conducted in Sigmo Health centre. Since knowing the prevalence of malaria is very important and the prevalence of malaria in Sigmo Health centre is yet not well known the investigator was interested to do research on this area to fill the knowledge gap. The main objective of this study is to assess the prevalence of malaria from 2000 to 2009, in the case of Sigmo health center, Jimma zone, Southwest Ethiopia. The study was conducted in Sigmo health centre, Sigmo woreda, located in Jimma zone from Dec 2016 to August 2017. The source of population was all patients admitted at Sigmo health center from 2000-2009. Secondary data collected from from the health centre was used as a source of data. After collected data cleared and checked for their completeness, the data were analyzed by using a scientific calculator. Accordingly compared to other areas, in the current study site, the prevalence of malaria was low (3\%) throughout the years (2000-2009). The most prevalent parasite in the study site was plasmodium vivax $(76 \%)$ and female were the most affected by malaria. Thus, the declining pattern of malaria infection in the study area could be evidence for intense intervention strategies undertaking in the study area.
\end{abstract}

Keywords: Malaria, Infection, Vectors, Prevalence

\section{Introduction}

Despite considerable efforts made throughout the century to eradicate or control malaria it is still the most prevalent and most devastating disease in the global tropics. The disease has a negative effect on the economic growth and perpetuates vicious cycles of poverty especially in the developing parts of the world. It costs Africa US\$10 - 12 billion every year in lost domestic product even though it could be controlled for a fraction of that sum. In Africa, the malaria causes about $20 \%$ of cerebral conditions leading to coma and death. One important strategy to prevent malaria infection is the use of insecticide treated mosquito net
(ITNs). The use of bed nets, especially the ITNs can reduce both transmission and mortality by at least $25 \%$ when used properly [1].

Malaria is the most prevalent human parasitosis at global level, and in the last seven years in Venezuela, 202,800 new malaria infections have been reported, originating mainly in Bolivar, Sucre and Amazonas States. The Amazonian region is characterized as a tropical rain forest with high precipitation, and high temperature and humidity throughout the year. This region is inhabited mainly by indigenous people, which have nomadic habits and rudimentary housing, making the application of control measures with insecticides and antimalarial drugs more difficult. Malaria infections are produced by $\mathrm{P}$. falciparum, $\mathrm{P}$. vivax and occasionally $\mathrm{P}$. 
malariae. The main vector reported for this area is A. darlingi, which shows relatively good tolerance but high irritability to the insecticides used in this region. This species has exophilic habits [2].

Malaria epidemiology is affected by the interaction among human (e.g. age, immunity, nutritional state) climatic (mainly temperature and humidity) and biological factors (vectors, parasites) which contribute to the establishment of the infection in endemic areas. The effects of each of these factors must be elucidated in order to implement effective control measures to reduce the prevalence of malaria infection in endemic areas [3].

The malaria transmission dynamics in the Kassena Nankana District, a site in northern Ghana proposed for testing malaria vaccines. Intensive mosquitoes sampling was done for one year using human landing catches in three micro-ecological sites that is irrigated, lowland and rocky highlands. Transmission was highly seasonal and the heaviest transmission occurred from June to October. The intensity of transmission was higher for people in the irrigated communities than the non-irrigated ones [4].

The Dega zone of Ethiopia (altitude above 2,500 meters) with a mean annual temperature of 10-15 degree Celsius are malaria-free. Much of the Woina Dega zone (Altitude 1500 2500 meters) is also malaria free, especially the zone in the 2000 - 2500 meters above sea level. Malaria in Ethiopia "...often occurs below 2000 meters, with short-lived transmission following the rains. However, malaria epidemics have been recorded up to 2400 meters during periods when increased temperature and adequate precipitation are conducive for both vector survival and parasite development within the vector" [5].

\subsection{Statement of the Problem}

Most people at risk of the disease live in areas of relatively stable malaria transmission - infection is common and occurs with sufficient frequency that some level of immunity develops. A smaller proportion of people live in areas where risk of malaria is more seasonal and less predictable, because of either altitude or rainfall patterns. People living in the peripheral areas north or south of the main endemic area are vulnerable to highly seasonal transmission and to malaria epidemics. In areas of stable malaria transmission, very young children and pregnant women are the population groups at highest risk for malaria morbidity and mortality [6].

Many researches were done in Ethiopia on the prevalence of malaria. Even though many researches were done on the prevalence of malaria in other place of the country, no any recent study conducted in Sigmo Health centre. Since knowing the prevalence of malaria is very important and the prevalence of malaria in Sigmo Health centre is yet not well known the investigator was interested to do research on this area to fill the knowledge gap.

Research Questions:

1) What is the level of prevalence of malaria at Sigmo health centre from 2000-2009?

2) What is malaria prevalence among different sex group?
3) What is the proportion of plasmodium species causing malaria in the study area?

\subsection{Objective}

\subsubsection{General Objective}

The general objective of this study is to assess the prevalence of malaria from 2000 to 2009 , in the case of Sigmo health center, jimma zone, south west Ethiopia

\subsubsection{Specific Objectives}

1) To assess the prevalence of malaria in Sigmo health centre from 2000-2009

2) To determine the proportion of plasmodium species causing malaria in the study area from 2000-2009 E.C.

3 ) To assess proportion of malaria patients with sex (male and female) from 2000-2009.

\subsection{Significance of the Study}

The prevalence of malaria should be known well because if the prevalence increases, it causes many people to death. But since its prevalence known and if known as the prevalence increase, it is possible to find solution or it is possible to eradicate the mosquito using different mosquito eradication mechanisms. To do this it is very important to know the level of malaria prevalence. To know the level of prevalence this study is very significant.

\section{Literature Review}

Climatic condition may influence mosquito breeding and hence transmission of malaria. Anopheles gambiae breed in temporary pools such as roadside ditches and drains, burrow pits, hoof prints, wheel nuts, and puddles and highly depend on the rains for their numbers. These species have the highest population density during the rainy season and these accounts for the high incidence of malaria at this period of the year. In the hot dry seasons, substantial number of people may sleep outside in the open air, as a consequence of being bitten more frequently by exophagic species of mosquitoes. During the wet and cold season the species multiply rapidly thus increase their population and biting frequency. The immunity in this framework is divided into two, that of the insect and man's natural resistance. Sometimes the vectors develop resistance to some of the control measures notably in the insecticides because of how these insecticides are used [7].

In humans, malaria is caused by four species of the plasmodium protozoa (single celled parasites) - plasmodium falciparum, plasmodium vivax, plasmodium ovale and plasmodium malariae. Of these species plasmodium falciparum accounts for the majority of infections and is the most lethal. In a parasitology laboratory, malaria was found to be the major killer of paediatric illness and death in Kinshasa [8].

The study in Ghana sought to compare household's data on acute morbidity and treatment seeking behaviour in two districts with the use of health facility data). Since several countries extend malaria treatment to include the community 
and the home through public and private, formal and informal sectors, the need for more comprehensive estimates becomes urgent [9].

Most recent epidemiological studies in endemic areas of malaria have been carried out with symptomatic patients and attempt to correlate the infection with epidemiological factors. The use of more precise diagnostic methods in population based studies, allowing for screening for both symptomatic and asymptomatic individuals taken at random from the population, could produce a better understanding of the multiple epidemiological factors affecting malaria infection [10].

Microscopic diagnosis consisted in the observation of the Plasmodium parasite in the blood of studied individuals seen under a microscope. Thick and thin smears were prepared from blood drops taken from one of the ear lobes of each individual, and immediately stained with Giemsa for 15 minutes. The slides were examined under a light microscope and the results were scored after the observation of 100 fields at $100 \mathrm{X}$ magnification. The diagnosis of the slides was carried out by a trained microscopist from the malaria control services located in the area. All individuals were immediately told the results of the microscopy diagnosis, and those who were diagnosed positive for any Plasmodium species received the corresponding treatmen [11].

Temperature and humidity have a direct effect on the longevity of the mosquito. Each species can thrive at an optimal level as a result of ecological adaptation. The spread of malaria requires that conditions are favorable for the survival of both the mosquito and the parasite. Temperatures from approximately $21^{\circ}-32^{\circ} \mathrm{C}$ and a relative humidity of at least $60 \%$ are most conducive for maintenance of transmission [12].

\subsection{The Ways of Mosquito Vector to find the Human Host}

Mosquitoes possess a highly developed set of hostdetection organs that they use to sense color, light, touch, and temperature as well as the presence of $\mathrm{CO} 2$. They use all of these sensory inputs to locate a host. When a mosquito has found an appropriate host on which to feed, it uses its two pairs of mandibles and maxillae to penetrate the skin of the animal and pierce a capillary vessel, allowing easy access to blood. It injects numerous biologically active compounds that cause the vessels to dilate, blood to flow without coagulation, and to prevent the host from detecting her presence [13].

\subsection{Malaria's Disease Burden in Ethiopia}

Malaria transmission peaks bi-annually from September to December and April to May coinciding with major harvesting season with serious $\mathrm{s}$ consequences for the subsistence Economy of Ethiopia's countryside, and for the nation in general. In addition, major epidemics occur every five to eight years with focal epidemics as the commonest form. The overall trend is one of a gradual progression from around four-fifths of a million confirmed cases a year in 1990 to almost a million and a half in the year 2005-06 reporting period. This represents an increase of about $80 \%$. The turn of the century (1999-00) marked the worst decade of malarial infections in recent history with the number of confirmed cases exceeding one and a half million a year [14].

Among the four plasmodium species known to cause malaria in Ethiopia, the two epidemiologically important species are Plasmodium falciparum and Plasmodium vivax comprising $60 \%$ and $40 \%$ respectively. Plasmodium falciparum is the cause of severe and complicated malaria which cause fatality rate of about $10 \%$ in hospitalized adults and up to $33 \%$ in children [13].

\section{Method and Material}

\subsection{The Study Area and Period}

The study was conducted in Sigmo health centre, Sigmo woreda is located in Jimma zone from Dec 2016 to August 2017. Jimma town is a capital of Jimma zone and located 356 $\mathrm{km}$ far from Addis Ababa Ethiopia.

\subsection{Study Design}

A retrospective study design was employed.

\subsection{Populations}

\subsubsection{Source Population}

The source of population was all patients admitted at Sigmo health center from 2000-2009. Secondary data was collected from the health center.

\subsubsection{Study Population}

The study population of this study was patients admitted at Sigmo health center for the case of malaria infection

\subsection{Sampling Techniques}

From the admitted patients at Sigmo health centre the document of all patients has been taken and those patients treated by the case of malaria infection were selected

\subsection{Data Collection Instrument}

Data were collected from documents of all patients admitted at Sigmo health centre from 2000 to 2009 E.c.

\subsection{Method of Data Analysis}

After collected data cleared and checked for their completeness, the data will be analyzed by using a scientific calculator and results will be presented in the form of table.

\subsection{Ethical Issue}

The study was ethically accepted by the administrator of Sigmo Health Center. All data were handled confidentially and ethically.

\section{Results}

\section{Prevalence of malaria}

The overall prevalence of malaria in the study area showed 
slightly lower. In all cases (2000-2009 E.C) the prevalence was lower than $3 \%$ (Table 1 ).

Table 1. Prevalence of malaria documented in Sigmo health centre from 2000-2009 E.C.

\begin{tabular}{llll}
\hline $\begin{array}{l}\text { Year } \\
\text { (E.C) }\end{array}$ & $\begin{array}{l}\text { Number of } \\
\text { examined cases }\end{array}$ & $\begin{array}{l}\text { Number of malaria } \\
\text { positive cases }\end{array}$ & $\begin{array}{l}\text { \%prevalence } \\
\text { of malaria }\end{array}$ \\
\hline 2000 & 6130 & 127 & 2.07 \\
2001 & 6210 & 169 & 2.72 \\
2002 & 6345 & 156 & 2.46 \\
2003 & 6001 & 140 & 2.33 \\
2004 & 5947 & 127 & 2.14 \\
2005 & 6161 & 111 & 1.8 \\
2006 & 6199 & 102 & 1.65 \\
2007 & 5967 & 89 & 1.49 \\
2008 & 5746 & 77 & 1.34 \\
2009 & 3610 & 69 & 1.91 \\
\hline
\end{tabular}

According to date obtained from the health center, number of malaria positive observed in year from 2000-2009 was different. It showed a decreasing pattern. The highest malaria positive individuals was observed in year $2001 \quad(n=169)$ followed by year $2002(n=156)$. Then with similar pattern the number decreased to 77 and 69 in year 2008 and 2009 respectively (Figure 1).
With respect of infection with different plasmodium species, both plasmodium vivax and $p$. falciparum are the common malaria parasite in the study area. However, throughout the years the dominant parasite is plasmodium vivax with proportion greater than $60 \%$. Even during 2008 and 2009, although the number of malaria positive patients less compared to other years, proportion of $p$. vivax infected patients was higher than those infected with $p$. falciparum (Table 2).

Table 2. Malaria prevalence with regard to different plasmodium species documented in Sigmo health center from 2000-2009.

\begin{tabular}{lll}
\hline Year (E.C) & P.vivax (\%) & p.falciparum (\%) \\
\hline 2000 & $82(62.57)$ & $45(35.43)$ \\
2001 & $100(59.17)$ & $69(40.83)$ \\
2002 & $101(64.74)$ & $55(35.26)$ \\
2003 & $97(69.29)$ & $43(30.71)$ \\
2004 & $84(66.14)$ & $43(33.86)$ \\
2005 & $70(63.06)$ & $41(36.94)$ \\
2006 & $63(61.76)$ & $39(38.24)$ \\
2007 & $53(59.55)$ & $36(40.45)$ \\
2008 & $45(58.44)$ & $32(41.56)$ \\
2009 & $42(60.87)$ & $27(39.13)$ \\
\hline
\end{tabular}

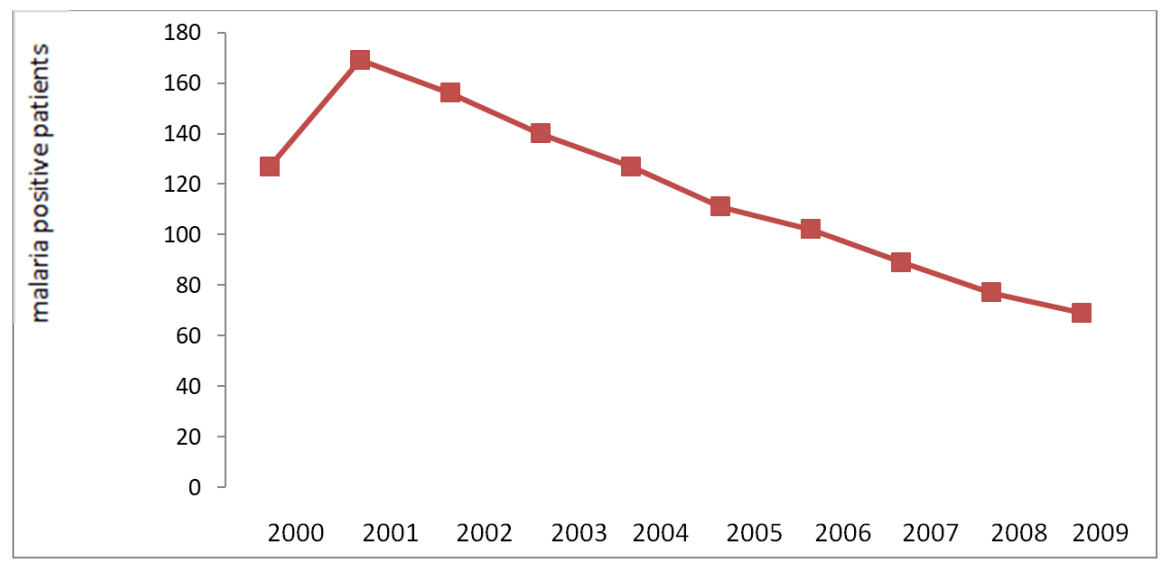

Figure 1. Number of malaria positive patients documented in Sigmo health center from 2000-2009 E.C.

According to the analysis made on the malaria positive patients seeking medication in the health center, number of female patients was higher than male. In some years (2000E.C) the proportion becomes even higher than $70 \%$ (72.44). In addition, in all years the number of female patients infected with malaria was higher than 55\% (Table 3).

Table 3. Prevalence of malaria among participants with different sexes in Sigmo health center from 2000-2009 E.C.

\begin{tabular}{lll}
\hline Year (E.C) & Male (\%) & Female (\%) \\
\hline 2000 & $37(29.13)$ & $92(72.44)$ \\
2001 & $66(39.05)$ & $103(60.95)$ \\
2002 & $61(39.1)$ & $95(60.9)$ \\
2003 & $57(40.71)$ & $83(59.29)$ \\
2004 & $51(40.16)$ & $76(59.84)$ \\
2005 & $49(44.14)$ & $62(55.86)$ \\
2006 & $43(42.16)$ & $59(57.84)$ \\
2007 & $37(41.57)$ & $52(58.43)$ \\
2008 & $31(40.26)$ & $46(59.74)$ \\
2009 & $26(37.68)$ & $43(62.32)$ \\
\hline
\end{tabular}

Table 4. Prevalence of malaria among different age groups in Sigmo health center from 2000-2009 E.C.

\begin{tabular}{lllll}
\hline S/N & Year (E.C) & $<$ 5 Years (\%) & 5-15 Years $(\%)$ & $\geq$ 15 Years $(\%)$ \\
\hline 1 & 2000 & $50(39.37)$ & $45(35.43)$ & $32(25.19)$ \\
2 & 2001 & $60(35.5)$ & $48(28.4)$ & $61(36.09)$ \\
3 & 2002 & $55(35.25)$ & $70(44.87)$ & $31(19.87)$ \\
4 & 2003 & $45(32.14)$ & $55(39.28)$ & $40(28.57)$ \\
5 & 2004 & $40(31.49)$ & $51(40.15)$ & $36(28.34)$ \\
6 & 2005 & $38(34.23)$ & $39(35.13)$ & $34(30.63)$ \\
7 & 2006 & $36(35.29)$ & $35(34.31)$ & $31(30.39)$ \\
8 & 2007 & $30(33.7)$ & $31(34.83)$ & $28(31.46)$ \\
9 & 2008 & $27(35)$ & $26(33.76)$ & $24(31.16)$ \\
10 & 2009 & $25(36.23)$ & $21(30.43)$ & $23(33.33)$ \\
\hline
\end{tabular}

According to data analysed, prevalence of malaria among different age groups of Sigmo woreda residents are different in each year from 2000-2009. Children less than five years were the most affected age group by malaria disease. For example in 2000 E.C about $50(39.37 \%)$ of the malaria patients were 
children less than five years. This is the most prevalent age group when compared to other from $5-15$ and $\geq 15$. This different prevalence of malaria according to the age group of patients similarly continues from 2000-2009 (Table 4).

\section{Discussion and Conclusion}

\subsection{Discussion}

According to data obtained, about 59.4\% males and $67.39 \%$ of females were admitted for the case of p.vivax. Whereas $40.5 \%$ of male and $32.61 \%$ were infected by p.falcparium and vesited Sigmo health centre. This is somewhat similar with the study conducted [13], among the four plasmodium species known to cause malaria in Ethiopia, the two epidemiologically important species are Plasmodium falciparum and Plasmodium vivax comprising $60 \%$ and $40 \%$ respectively. Plasmodium falciparum is the cause of severe and complicated malaria which cause fatality rate of about $10 \%$ in hospitalized adults and up to $33 \%$ in children.

Starting from 2000-2009 E.C the number of patients infected by p.falsiparium and p.vivax were uniformly become decreased. This may be due to decrement of number of vector anophline mosquito from the district or there may be decrement of infectivity rate of the mosquito. Not only this according to the documented data gained from Sigmo health centre, the number of patients or the disease prevalence was seasonal. For example, from September to November the number of patients documented each year were very high than in the other months of the years.

This is in agreement with the study conducted [7] Climatic condition may influence mosquito breeding and hence transmission of malaria. Anopheles gambiae breed in temporary pools such as roadside ditches and drains, burrow pits, hoof prints, wheel nuts, and puddles and highly depend on the rains for their numbers. These species have the highest population density during the rainy season and these accounts for the high incidence of malaria at this period of the year. In the hot dry seasons, substantial number of people may sleep outside in the open air, as a consequence of being bitten more frequently by exophagic species of mosquitoes. During the wet and cold season the species multiply rapidly thus increase their population and biting frequency. The immunity in this framework is divided into two, that of the insect and man's natural resistance. Sometimes the vectors develop resistance to some of the control measures notably in the insecticides because of how these insecticides are used.

As the data gained and analyses from Sigmo health center shows, malaria was more prevalent in females than males. For example, in 2000 E.C the prevalence of malaria was 37(29.13) and 92(72.44) in males and females respectively. While in 2001 E.C. the prevalence of this disease was increased to 66(39.05) and 103(60.95) in male and female respectively. This may be due to the lower immunity of females than males. This is similar with the study conducted $[6,15]$ in areas of stable malaria transmission, very young children and pregnant women are the population groups at highest risk for malaria morbidity and mortality.

As data analyses and compared the prevalence of malaria at Sigmo Woreda seems higher in year 2001 and 2002 this could be happened may be due to variation of weather condition. When the climate condition changes, the speed of mosquito breeding varies. So, the reason why the prevalence of malaria was increased in 2001 and 2002 was change of weather condition of Sigmo Woreda to very favourable condition to mosquito breeding. This is in agreement with one study found in $[12,15]$ temperature and humidity have a direct effect on the longevity of the mosquito. Each species can thrive at an optimal level as a result of ecological adaptation. The spread of malaria requires that conditions are favorable for the survival of both the mosquito and the parasite. Temperatures from approximately $21^{\circ}-32^{\circ} \mathrm{C}$ and a relative humidity of at least $60 \%$ are most conducive for maintenance of transmission

According to the age group the data showed as malaria was more prevalent in children less than five ages this may be due to favorable age condition to the parasite because of this most of the time the prevalence of malaria is age dependent. This result can be supported by the result of study conducted [6] People living in the peripheral areas north or south of the main endemic area are vulnerable to highly seasonal transmission and to malaria epidemics. In areas of stable malaria transmission, very young children and pregnant women are the population groups at highest risk for malaria morbidity and mortality.

\subsection{Conclusion}

The number of patients caused by p.falciparium and p.vivax were uniformly become decreased in the last 10 years. This may be due to decrement of number of vector anophline mosquito from the district or there may be decrement of infectivity rate of the mosquito. Not only this according to the documented data gained from Sigmo health centre, the number of patients or the disease prevalence was seasonal. For example, from September to November the number of patients documented each year were very high than in the other months of the years. This is due to the favourable condition to mosquito breeding was fulfilled during this season. For mosquito to breed themselves there should be wet or water which supports hatchment of eggs and development of larva and pupae. Because of this malaria was very prevalent at most each year from September to November in Sigmo district. As well as plasmodium vivax was the most prevalent malaria at Sigmo district in the last 10 years.

\section{Recommendation}

1) According to the data analysed showed, the prevalence of malaria in Sigmo woreda highly increase starting from September. Bearing this in mind everybody should keep his/her self from biting by mosquitoes by using mosquito prevention and control mechanisms such as bed net, biological control making bright in bed room at night. 
2) According to the result of this study, children less than five years old were highly malaria affected age group; So that, any concerned body should work to reduce the prevalence of this disease among children less than five years.

3) Even though the prevalence of this disease is different between male and female, among age groups and from season to season the data analyzed and presented in the result part of this study showed as the prevalence of this disease becomes decreased in Sigmo Woreda from 2000-2009, Since this is very nice hope full result, the retardation or the declination the disease prevalence should be continued up to the disappearance of the disease from the Sigmo woreda.

\section{References}

[1] Songsore. J., (2003). "The Urban Housing Crisis in Ghana: Capital, the state Versus the People", in Ghana Social Science Journal, Vol 2 No. 1, p4.

[2] ACHÉ, A.; ESCORIHUELA, M.; PÁEZ, E. (2002). In vivo drug resistance of falciparum malaria in mining areas of Venezuela. Trop. Med. Int. Hlth, 7: 737-743.

[3] ASLAN, G.; ULUKANLIGIL, M.; SEYREK, A. \& OZCAN, E. (2001). Diagnostic performance characteristics of rapid dipstick test for Plasmodium vivax malaria. Mem. Inst. Oswaldo Cruz, 95: 683-686.

[4] Apawu. M. (2004). "Malaria Transmission Dynamics At A Site In Northern Ghana Proposed For Testing Malaria Vaccine" in Tropical Medicine for International Health. pp. 160-70.
[5] Tedros Adhanom, Malaria. in Yemane Berhane, (eds, (2006). Epidemiology and Ecology of Health and Disease in Ethiopia. Shama books. Addis Ababa.

[6] The World Health Report (2002). Reducing risks, promoting healthy life. Geneva, World Health Organization.

[7] MAKLER, M. T.; PALMER, C. J. \& AGER, A. L. (1998). A review of practical techniques for the diagnosis of malaria. Ann. trop. Med. Parasit., 92: 419-433.

[8] Coene. J., (1991). "Prospects for Malaria Control in Urban and Rural Kinshasa", Ann Soc Belg Med Trop, p71.

[9] Agyapong. I. A. \& Kangeya-Kayonda. J. (2004). "Providing Practical Estimates of Malaria Burden for Health Planners in Resource-Poor Countries", in American Journal of Tropical medicine and Hygiene, pp. 162-7.

[10] Service. M. W., (1979). "Introduction to the Mosquitoes" in A Guide To Medical Entomlogy. pp. 23-43.

[11] MILLER, L.; BARUCH, D.; MARSH, K. \& DOUMBO, O. K. (2002). The pathogenic basis of malaria. Nature, 415: 673679 .

[12] (http://www.medicalecology.org/diseases/malaria/print_malari a.htm\#sect5.2 updated: August, 2017).

[13] Federal Ministry of Health (FMH) (2004). Malaria: Diagnosis and Treatment guide line for Health Workers in Ethiopia. Addis Ababa, Ethiopia: Federal democratic Republic of Ethiopia, Ministry of Health; 2004.

[14] Chibsa, S. (2007). Malaria Vector Control Efforts and Challenges in Ethiopia. $4^{\text {th }}$ WIN meeting, Basel Switzerland.

[15] World Health Organization. Malaria Entomology and Vector Control (Learner's Guide). Geneva: WHO, 2003. 